\title{
EDITORIAL
}

\section{Why should we target viral serine proteases when developing antiviral agents?}

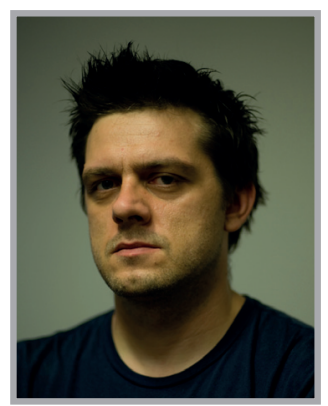

\author{
"The design of specific and selective inhibitors of \\ viral serine proteases may result in the \\ development of new powerful therapeutics...”
}

\section{Marcin Skoreński', Renata Grzywa' \& Marcin Sieńczyk*,1}

First draft submitted: 10 October 2016; Accepted for publication: 11 October 2016; Published online: 21 November 2016

The development of antiviral agents goes back to the 1950s when idoxuridine, the first antiviral drug, was discovered and approved in 1963 for the treatment of herpes simplex type 1 infection. Since then, many antiviral drugs have been developed and introduced for clinical use, targeting a particular pathogen (e.g., HIV, HCV) or displaying a broad spectrum of antiviral activity (e.g., ribavirin). For the first few decades, the drug development efforts were mainly focused on substances targeting the viral genome replication process with only few examples of drugs designed to act as entry inhibitors (VZIG influenza virus matrix protein 2 inhibitor (amantadine), pegylated interferons or antimitotic drug, podofilox. The situation changed in the 1990s mainly due to the growing threat of HIV/AIDS pandemic and the emerging risk associated with $\mathrm{HCV}$ discovered in 1989. In addition, failure to develop an effective vaccine against lethal viruses highlighted the necessity for searching new treatment options. As a result, several viral molecular targets were identified and used for the development of novel classes of antiviral drugs, including protease inhibitors (PIs) and integrase as well as HCV NS5A and NS5B inhibitors [1]. The introduction of PIs into HIV/AIDS therapy significantly reduced the mortality rate among AIDS patients and proved the importance of structure-based drug design approach as well as the significance of viral proteolytic enzymes as drug targets [2].

The successful therapeutic approach based on HIV aspartic protease inhibition was subsequently applied for the development of antivirals targeting HCV serine protease (NS3/4A). Similar to other viral proteases, HCV NS3/4A is responsible for processing viral polyprotein and therefore represents a key element in the virus replication cycle. As in the case of HIV protease, the rational design of NS3/4A inhibitors was triggered by the determination of protease crystal structure [3]. The first studies on HCV NS3/4A inhibitors led to the development of ciluprevir (BILN-2061). Unfortunately, ciluprevir failed the clinical trials on animals as it

\section{KEYWORDS}

- antiviral agents $\bullet$ drug design

- enzyme inhibitors $\bullet$ viral proteases

'Faculty of Chemistry, Division of Medicinal Chemistry \& Microbiology, Wroclaw University of Science \& Technology, Wybrzeze Wyspianskiego 27, 50-370 Wroclaw, Poland

*Author for correspondence: Tel.: +48 713203646; marcin.sienczyk@pwr.edu.pl

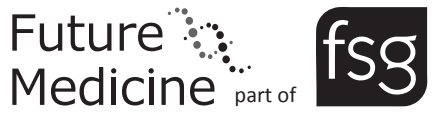


“Among already reported flaviviral protease

inhibitors, the most potent are basic and highly charged molecules...” proved to be toxic [4]. Nevertheless, this example served as an important proof of concept for PIs as useful anti-HCV agents. Just a few years later, boceprevir and telaprevir were approved by the US FDA as the first PIs for HCV treatment in a triple therapy with pegylated interferon and ribavirin. Until now, several first- and second-generation PIs used in combination with ribavirin or with $\mathrm{NS} 5 \mathrm{~B} / \mathrm{NS} 5 \mathrm{~A}$ protein inhibitors have been approved to treat $\mathrm{HCV}$-infected patients [5].

Although the introduction of PIs into antiviral therapy significantly improved its effectiveness, several limitations associated with PI-based therapies are yet to be overcome. Among the most important is a narrow virus genotype specificity of drugs and an increasing drug resistance of newly appearing mutant strains [6]. This can be explained not only by the differences in protease structure per se but also by the mechanism of viral proteases substrate recognition. Even the optimal substrate sequence can be determined with synthetic substrates, the studies on HIV as well as on HCV proteases have shown that the substrate recognition motif does not depend on the amino acid arrangement but rather on the structural 'shape' common to enzyme endogenous substrates called substrate envelope. Such a mechanism of recognition can explain the resistance of viral proteases to already developed drugs. Some mutations located at the protease substrate binding site could lead to weakening its interaction with the drug molecule without loss of overall substrate specificity [7]. Even though the substrate envelope recognition and associated drug resistance stunt the effective inhibitors' development, at the same time the drug structure design is not limited to a particular amino acid sequence, which is especially important for host proteases of specificity overlapping with viral enzymes. As an effective vaccine is still unavailable, the rational drug design and its combination with other substances is currently the most common approach used for $\mathrm{HCV}$ treatment where NS3/4A protease together with NS5A and NS5B remain the most important targets for drugs development [8].

Until now, the HCV NS3/4A is the only viral serine protease used as a drug target in clinical practice. The global threat posed by the recent appearance of the Zika virus (ZIKV) might accelerate the development of novel antiviral drugs targeting viral serine protease since the virus encodes a serine protease crucial for its lifecycle. The ZIKV belongs to the same family (Faviviridae) as the HCV but a different genus (Flavivirus) and shares significant sequence similarity to west nile virus (WNV), yellow fever virus (YFV) and dengue virus (DENV) [9]. Epidemiological reports indicate the connection of ZIKV infection with serious pathological disorders including microcephaly in newborns and Guillain-Barré syndrome in adults. Moreover, the virus may be transmitted not only by mosquitoes as initially suspected but also through sexual contact or blood [10]. Similar to other flaviviruses, ZIKV encodes a serine protease (NS2B-NS3) responsible for viral preprotein processing. As the crystal structure [11] and substrate specificity profile [12] of ZIKV NS2B-NS3 protease have already been determined, it might become the potential target for rational drug design. Since sequence homology and substrate recognition pattern display a significant similarity among flaviviruses, it is possible to design an inhibitor with a wide spectrum of antiviral activity.

Although several research groups have already identified a number of peptidic and nonpeptidic inhibitors of flaviviral proteases, the overall progress toward the selection of novel drug candidates has been unsatisfactory, placing flaviviral infections into the group of orphan diseases. One of the reasons is limited financing and human resources invested in the study. On the other hand, highly hydrophilic substrate binding sites of flavivirus proteases limit drug accessibility and the design of compounds displaying high potency, bioavailability and required pharmacological profile is highly challenging. In contrast to flaviviral serine proteases, the active site of HCV protease is less polar and more hydrophobic, which allows for developing drug candidates containing no hydrophilic or protonated substituents [13]. Among already reported flaviviral protease inhibitors, the most potent are basic and highly charged molecules and these could hinder membrane permeability and oral absorption. One of the solutions is the design of allosteric inhibitors that do not target the protease active site [14]. Such an approach could facilitate/accelerate the development of NS2B-NS3 protease inhibitors which could be found effective in ZIKV infections.

Another virus family encoding serine protease crucial for viral replication cycle includes herpesviruses. The activity of viral protease is key at the last stage of the virus lifecycle and 
is responsible for a successful capsid assembly and the viral DNA packing. The protease degrades the protein capsid scaffold leading to its collapse and thus making space for the viral DNA molecule [15]. Since the analysis of available crystal structures of herpesvirus serine proteases has provided details of the substrate binding sites, the design of potent and specific inhibitors should be feasible. Moreover, based on the homology and substrate recognition pattern between different proteases of different herpesviruses, the design of a compound with a broad-spectrum activity should also be possible. Unfortunately, despite many efforts to develop potent inhibitors of herpesvirus proteases, there is no single approved drug or even a compound in clinical trials that displays such a mechanism of action [16]. The development of herpesvirus PIs is challenging due to several aspects. One is the presence of a noncanonical catalytic apparatus composed of serine residue (Ser132) responsible for the nucleophilic attack on the carbonyl carbon of the scissile peptide bond and two histidine residues (His63 and His157) [17]. Such an unusual catalytic triad due to its lower catalytic activity might be less susceptible to inhibition. Additionally, herpesvirus proteases exist in a concentration-dependent monomer-dimer equilibrium state while only dimeric form is catalytically active [18]. This monomer-dimer equilibrium could represent another limitation for an effective inhibitors development. It has been shown that substrate/inhibitor binding promotes enzyme dimerization; however, it is not clear whether binding occurs to a monomer promoting dimerization or the substrate/inhibitor binds only to the dimer stabilizing this state [19]. If the inhibitor is able to bind only to the dimeric form of protease that exists only inside the premature capsid in the cell nucleus, it could be difficult to achieve required inhibitor concentration in the nucleus. On the other hand, if the inhibitor is meant to be bound by an inactive monomer, the structure of the inhibitor needs to be optimized in order to allow its binding to the inactive center of the protease present in monomeric form as well as the binding site of an active dimer. Such a requirement is difficult to fulfill by inhibitors containing a warhead forming a covalent bond with the active site serine. The development of allosteric inhibitors which disrupt or block the dimerization process is one of the solutions for overcoming these problems [20].

The design of specific and selective inhibitors of viral serine proteases may result in the development of new powerful therapeutics. The proof of the concept of this approach is represented by the successful FDA-approved anti-HCV drugs whose mode of action relies on the inhibition of viral serine protease, a precise molecular scissors that trim proteins important for the virus to become infectious. Unfortunately, similar studies on the development of new antiflavivirus (e.g., WNV, DENV, YFV, ZIKV) and antiherpesvirus (e.g., HSV-1, HSV-2, CMV, varicella zoster virus) agents are less advanced $[13,16]$. One challenge in the design of inhibitors is their limited activity in cell assays as compared with their promising activity in enzymatic assays. Nevertheless, being an essential element of viral replication, the serine proteases represent an excellent target for rational drug design.

In the light of many years of significant failure of the development of effective vaccine toward highly dangerous viruses, the design of low-molecular weight inhibitors and selection of highly active drugs among them seems like the best case scenario. In addition, considering the rapid spread of ZIKV infections worldwide, the global scientific effort might bring the new solution sooner than one can expect.

\section{Financial \& competing interests disclosure}

$M$ Sieńczyk and R Grzywa would like to thank the Polish Ministry of Science and Higher Education for subsidiary founds (S50129/Z0313). The work was partially supported by the Polish Ministry of Science and Higher Education Iuventus Plus Program (IP2012 0556 72). The authors have no other relevant affliations or financial involvement with any organization or entity with a financial interest in or financial conflict with the subject matter or materials discussed in the manuscript apart from those disclosed.

No writing assistance was utilized in the production of this manuscript

\section{References}

Papers of special note have been highlighted as:

- of interest; $\bullet$ of considerable interest

1 De Clercq E, Li G. Approved antiviral drugs over the past 50 years. Clin. Microbiol. Rev. 29, 695-747 (2016).
-. Comprehensive review on approved antiviral drugs developed over the last 50 years.

2 SkoreŃski M, SieŃczyk M. Viral proteases as targets for drug design. Curr. Pharm. Des. 19, 1126-1153 (2013).
3 Kim JL, Morgenstern KA, Lin C et al. Crystal structure of the hepatitis C virus NS3 protease domain complexed with a synthetic NS4A cofactor peptide. Cell 87, 343-355 (1996).

- Reports the crystal structure of HCV NS3/4A protease. 
4 Lamarre D, Anderson PC, Bailey M et al. An NS3 protease inhibitor with antiviral effects in humans infected with hepatitis $\mathrm{C}$ virus. Nature 426, 186-189 (2003).

5 De Luca A, Bianco C, Rossetti B. Treatment of HCV infection with the novel NS3/4A protease inhibitors. Curr. Opin. Pharmacol. 18, 9-17 (2014).

6 Kieffer TL, George S. Resistance to hepatitis C virus protease inhibitors. Curr. Opin. Virol. 8, 16-21 (2014).

7 Kurt Yilmaz N, Swanstrom R, Schiffer CA. Improving viral protease inhibitors to counter drug resistance. Trends Microbiol. 24, 547-57 (2016).

-• Excellent review on the subject of viral protease drug resistance mechanisms and the strategies of protease inhibitors drug design.

8 Zhang X. Direct anti-HCV agents. Acta Pharm. Sin. B 6, 26-31 (2016).

9 Weaver SC, Costa F, Garcia-Blanco MA et al. Zika virus: history, emergence, biology, and prospects for control. Antiviral Res. 130, 69-80 (2016).
- Excellent review that discusses the Zika virus infection.

10 Ticconi C, Pietropolli A, Rezza G. Zika virus infection and pregnancy: what we do and do not know. Pathog. Glob. Health 30, 1-7 (2016).

11 Lei J, Hansen G, Nitsche C, Klein CD, Zhang L, Hilgenfeld R. Crystal structure of Zika virus NS2B-NS3 protease in complex with a boronate inhibitor. Science 353, 503-505 (2016).

- Reports the crystal structure of Zika virus NS2B-NS3 protease.

12 Gruba N, Rodriguez Martinez JI, Grzywa R et al. Substrate profiling of Zika virus NS2B-NS3 protease. FEBS Lett. doi:10.1002/1873-3468.12443 (2016) (Epub ahead of print).

13 Poulsen A, Kang C, Keller TH. Drug design for flavivirus proteases: what are we missing? Curr. Pharm. Des. 20, 3422-3427 (2014).

14 Yildiz M, Ghosh S, Bell JA, Sherman W, Hardy JA. Allosteric inhibition of the NS2B-NS3 protease from dengue virus. ACS Chem. Biol. 8, 2744-2752 (2013).
15 Borthwick AD. Design of translactam HCMV protease inhibitors as potent antivirals. Med. Res. Rev. 25, 427-452 (2005).

16 Skorenski M, Sienczyk M. Anti-herpesvirus agents: a patent and literature review (2003 to present). Expert Opin. Ther. Pat. 24(8), 925-941 (2014).

17 Hoog SS, Smith WW, Qui X et al. Active site cavity of herpesvirus proteases revealed by the crystal structure of herpes simplex virus protease/inhibitor complex. Biochemistry 36(46), 14023-14029 (1997).

18 Schmidt U, Darke PL. Dimerization and activation of the herpes simplex virus type 1 protease. J. Biol. Chem. 272, 7732-7735 (1997).

19 Gable JE, Acker TM, Craik CS. Current and potential treatments for ubiquitous but neglected herpesvirus infections. Chem. Rev. 114(22), 11382-11412 (2014).

- Comprehensive review on development of antiherpesvirus agents.

20 Lee GM, Shahian T, Baharuddin A, Gable JE, Craik CS. Enzyme inhibition by allosteric capture of an inactive conformation. J. Mol. Biol. 411(5), 999-1016 (2011). 